\title{
Adjunctive Efficacy of Intra-Arterial Conebeam CT Angiography Relative to DSA in the Diagnosis and Surgical Planning of Micro-Arteriovenous Malformations
}

\author{
(D) A.S. Al-Smadi, (D) A. Elmokadem, (D)A. Shaibani, (DM.C. Hurley, (D) M.B. Potts, DB.S. Jahromi, and (D).A. Ansari
}

\begin{abstract}
BACKGROUND AND PURPOSE: Micro-arteriovenous malformations are an underrecognized etiology of intracranial hemorrhage. Our study aimed to assess the adjunctive efficacy of intra-arterial conebeam CTA relative to DSA in the diagnosis and surgical planning of intracranial micro-AVMs.

MATERIALS AND METHODS: We performed a retrospective study of all micro-AVMs ( $\leq 1-\mathrm{cm}$ nidus) at our institution. Blinded neuroradiologists qualitatively graded DSA and intra-arterial conebeam CTA images for the detection of specific micro-AVM anatomic parameters (arterial feeder, micronidus, and venous drainer) and defined an overall diagnostic value. Statistical and absolute differences in the overall diagnostic values defined the relative intra-arterial conebeam CTA diagnostic values, respectively. Blinded neurosurgeons reported their treatment approach after DSA and graded the adjunctive value of intra-arterial conebeam CTA to improve or modify treatment. Intraarterial conebeam CTA efficacy was defined as interobserver agreement in the relative intra-arterial conebeam CTA diagnostic and/or treatment-planning value scores.
\end{abstract}

RESULTS: Ten patients with micro-AVMs presented with neurologic deficits and/or intracranial hemorrhages. Both neuroradiologists assigned a higher overall intra-arterial conebeam CTA diagnostic value $(P<.05)$, secondary to improved evaluation of both arterial feeders and the micronidus, with good interobserver agreement $(\tau=0.66, P=.018)$ in the relative intra-arterial conebeam CTA diagnostic value. Both neurosurgeons reported that integrating the intra-arterial conebeam CTA data into their treatment plan would allow more confident localization for surgical/radiation treatment (8/10; altering the treatment plan in 1 patient), with good interobserver agreement in the relative intra-arterial conebeam CTA treatment planning value $(\tau=0.73, P=.025)$.

CONCLUSIONS: Adjunctive intra-arterial conebeam CTA techniques are more effective in the diagnostic identification and anatomic delineation of micro-AVMs, relative to DSA alone, with the potential to improve microsurgical or radiosurgery treatment planning.

ABBREVIATIONS: mAVM = micro-AVM; IA-CBCTA = intra-arterial conebeam CTA; 3DRA = 3D rotational angiography

C erebral micro-arteriovenous malformations (mAVMs) are a rare subgroup, accounting for $\sim 8 \%$ of intracranial AVMs in the surgical series by Stiver and Ogilvy ${ }^{1}$ and may represent $21 \%$ of AVMs presenting with intracranial hemorrhage in young adults. ${ }^{1,2}$ In 2013, Alén et $\mathrm{al}^{3}$ reported approximately $87 \%$ of patients with $\mathrm{mAVMs}$ presenting with spontaneous hemorrhage, while previous studies reported a 100\% incidence. ${ }^{2,4,5}$ Yasargil

Received February 15, 2018; accepted after revision June 12.

From the Departments of Radiology (A.S.A.-S., A.E., A.S., M.C.H., M.B.P., B.S.J., S.A.A.), Neurology (S.A.A.), and Neurological Surgery (A.S., M.C.H., M.B.P., B.S.J., S.A.A.), Northwestern University, Feinberg School of Medicine, Chicago, Illinois.

Please address correspondence to Sameer A. Ansari, MD, PhD, Departments of Radiology, Neurology, and Neurological Surgery, Northwestern University, Feinberg School of Medicine, 676 N St. Clair St, Suite 800, Chicago, IL 60611-2927; e-mail: s-ansari@northwestern.edu

三 Indicates article with supplemental on-line tables.

http://dx.doi.org/10.3174/ajnr.A5745 defined $m A V M s$ as a particular entity of pial AVMs characterized by an occult nidus of $\leq 1 \mathrm{~cm}$ and differentiated this subgroup, which could not be visualized on angiography or gross pathology specimens but could be identified on histopathology if the hematoma was carefully removed. ${ }^{6}$

Micro-AVMs may be suspected on cerebral DSA as an abnormal draining vein with variable detection of a feeding artery and/or micronidus, often only suggested by small coalescing arterioles. Subtle angiographic findings make the imaging diagnosis challenging and broaden the differential diagnosis to include the possibility of a dural or pial arteriovenous fistula. Additionally, the small $\mathrm{mAVM}$ size and associated hemorrhage add difficulty in lesion localization for treatment planning with either microsurgical resection or stereotactic radiation therapy.

Digital flat panel detector CT technology has improved the imaging capabilities of modern angiographic equipment with the 
feasibility of routinely performing $3 \mathrm{D}$ rotational angiography (3DRA) and intra-arterial conebeam CTA (IA-CBCTA). IACBCTA incorporates the high spatial vascular resolution of 3DRA with CT postprocessing techniques for increased contrast resolution to visualize the peripheral osseous and soft tissues. High spatial resolution is maintained and displayed in submillimeter multiplanar reconstructions for improved diagnostic sensitivity and cross-sectional mapping. ${ }^{7}$ Prior studies have described the efficacy of IA-CBCTA for the anatomic localization of intracranial and spinal dural AVFs. ${ }^{8,9}$ In this study, we hypothesized that the efficacy of IA-CBCTA relative to DSA would improve the diagnostic identification, localization, and treatment planning of mAVMs.

\section{MATERIALS AND METHODS \\ Patient Study}

Institutional review board approval was obtained for a retrospective study of patients presenting with intracranial mAVMs $(\leq 1$ $\mathrm{cm}$ ) between January 2010 and February 2017. All patients were identified through the neuroangiographic database of our institution, and at least 1 pretreatment DSA study with adjunctive IA-CBCTA imaging was required for study inclusion. The clinical and radiologic findings of these patients and $\mathrm{mAVM}$ lesions were reviewed, including patient demographics, presentations, intracranial hemorrhage locations and volumes (gross manual calculations on presenting CT/MR imaging), ${ }^{10} \mathrm{mAVM}$ classification according to the Spetzler-Martin grading system, and subsequent management/treatment.

\section{Image Acquisition}

Biplane DSA imaging studies were acquired at 2 tertiary academic institutions (Northwestern Memorial Hospital and Lurie Children's Hospital affiliated with Northwestern University, Chicago, Illinois) with identical biplane angiography suites (Artis Zee/ Zeego flat detector biplane angiosuite; Siemens, Erlangen, Germany) and acquisition protocols. 3DRA or IA-CBCTA scanning was performed with a single flat panel detector rotational fluoroscopy and angiography unit ( $2 \mathrm{k}$ detector, amorphous silicon cesium iodide scintillator; $1920 \times 2480$ pixel resolution, $3.25 \mathrm{lp} /$ $\mathrm{mm}$; size, $30 \times 40 \mathrm{~cm}$ ). The motorized frontal C-arm was used to acquire 496 projection frames over a $200^{\circ}$ arc with 5 - to 8 -second rotation times at $80 \mathrm{kV}$ and $260 \mathrm{~mA}$ and a radiation dose of 1.2 $\mu \mathrm{Gy} /$ frame. Although acquisition parameters remained constant, IA-CBCTA techniques varied slightly with respect to intra-arterial contrast injection rates dependent on vessel injection sites, including the internal carotid artery $(4 \mathrm{~mL} / \mathrm{s})$ and vertebral artery (3-4 mL/s), with total volumes of 15-32 mL. Shorter acquisition times (5-8 seconds) and nondilute iodinated contrast assisted with opacification of subtle microvascular anatomy and abnormal early venous drainage without venous contamination.

IA-CBCTA acquisition data were transferred to an independent 3D postprocessing workstation (Leonardo; Siemens). Multiplanar, subtracted, and unsubtracted CT reconstructions (sagittal, coronal, and axial planes with overlapping $0.5-\mathrm{mm}$ slice thickness) were generated and transferred to a PACS for direct visualization on the PACS workstation.

\section{Image Analysis}

Images were retrospectively and independently reviewed by 2 interventional neuroradiologists and 2 vascular neurosurgeons on a de-identified PACS system (including the postprocessed multiplanar IA-CBCTA reconstructions). Both neuroradiologists graded the DSA (biplane, magnified, oblique views) and 3DRA images without access to the IA-CBCTA dataset. Subsequent IACBCTA images (subtracted/unsubtracted multiplanar reconstructions) acquired from the same intra-arterial injection site were scored by the observers. Qualitative image analyses were performed on high-definition liquid crystal display monitors routinely used for diagnostic reporting and, based on the level of image quality, anatomic and angiographic characterization on a scale of $0-2$ (2, excellent/good; 1 , relevant visibility with restrictions; 0 , poor, nondiagnostic). The reviewers scored the following parameters: 1) arterial feeders (anatomic localization, origin/course, single versus multiple); 2) venous drainers (anatomic localization, course/destination, single versus multiple); and 3) nidus site (anatomic localization, size, and differentiation from arteriovenous shunting). The total score for each technique was defined as the overall diagnostic value for interpretation.

For evaluation of treatment-planning efficacy, the 2 vascular neurosurgeons reported their presumed treatment strategy and approach (if applicable) after evaluating DSA and 3DRA imaging and subsequently studied the IA-CBCTA multiplanar reconstructions. Both neurosurgeons then graded the adjunctive or relative treatment-planning values of IA-CBCTA according to a scale of $0-2$ (2, altered treatment plan; 1 , more confident treatment plan; or 0 , no value). They were required to provide a reason if they determined that IA-CBCTA improved or altered the treatment plan (grade 1 or 2) in comparison with DSA imaging alone.

\section{Statistical Analysis}

For each observer, the Wilcoxon test was used to assess statistical differences in DSA-versus-IA-CBCTA scoring for each of the 3 $\mathrm{mAVM}$ anatomic parameters and the overall diagnostic values (sum of all 3 parameter scores) between the 2 modalities. Statistical and absolute differences between the overall IA-CBCTA and DSA diagnostic value defined the relative IA-CBCTA diagnostic value/scores, respectively. A $P$ value $<.05$ was considered a statistically significant difference.

Interobserver agreement for DSA and IA-CBCTA scoring of each $\mathrm{mAVM}$ parameter and the overall diagnostic values were assessed using the Kendall $\tau$ coefficient. Interobserver agreement of the relative IA-CBCTA diagnostic value scores (absolute difference between overall IA-CBCTA and DSA diagnostic value scores), and relative IA-CBCTA treatmentplanning value scores were also evaluated using the Kendall $\tau$ coefficient. Good or excellent agreement defined the efficacy of IA-CBCTA relative to DSA in the diagnosis and surgical planning of mAVMs, respectively. The $\tau$ coefficient varied between 0 and 1 , with 0 representing no agreement and 1 representing complete agreement. $\tau$ values of $>0.8,>0.5-0.8,>0.2-0.5$, and $\leq 0.2$ were considered to indicate excellent, good, fair, and poor agreement, respectively. A $P$ value $<.05$ indicated statis- 
Table 1: Qualitative diagnostic scoring of DSA and IA-CBCTA imaging

\begin{tabular}{|c|c|c|c|c|c|c|c|c|c|c|c|c|c|c|c|c|c|c|}
\hline & \multicolumn{9}{|c|}{ Observer 1} & \multicolumn{9}{|c|}{ Observer 2} \\
\hline & \multicolumn{2}{|c|}{$\begin{array}{l}\text { Arterial } \\
\text { Feeder }\end{array}$} & \multicolumn{2}{|c|}{ Nidus } & \multicolumn{2}{|c|}{$\begin{array}{l}\text { Venous } \\
\text { Drainage }\end{array}$} & \multicolumn{2}{|c|}{ Overall } & \multirow{2}{*}{$\begin{array}{c}\text { Relative } \\
\text { CBCTA } \\
\text { Diagnostic } \\
\text { Value }^{\mathrm{a}}\end{array}$} & \multicolumn{2}{|c|}{$\begin{array}{l}\text { Arterial } \\
\text { Feeder }\end{array}$} & \multicolumn{2}{|c|}{ Nidus } & \multicolumn{2}{|c|}{$\begin{array}{l}\text { Venous } \\
\text { Drainage }\end{array}$} & \multicolumn{2}{|c|}{ Overall } & \multirow{2}{*}{$\begin{array}{c}\text { Relative } \\
\text { CBCTA } \\
\text { Diagnostic }_{\text {Value }}{ }^{\mathrm{a}}\end{array}$} \\
\hline & DSA & СВСТА & DSA & СВCTA & DSA & СВCTA & DSA & СВCTA & & DSA & СВСТА & DSA & СВСТА & DSA & СВCTA & DSA & CBCTA & \\
\hline 1 & 1 & 2 & 0 & 2 & 2 & 2 & 3 & 6 & 3 & 0 & 2 & 0 & 2 & 2 & 2 & 2 & 6 & 4 \\
\hline 2 & 0 & 2 & 1 & 2 & 2 & 2 & 2 & 6 & 4 & 0 & 2 & 1 & 2 & 2 & 2 & 3 & 6 & 3 \\
\hline 3 & 1 & 2 & 1 & 2 & 2 & 2 & 4 & 6 & 2 & 0 & 2 & 1 & 2 & 2 & 2 & 3 & 6 & 3 \\
\hline 4 & 1 & 2 & 0 & 2 & 1 & 2 & 2 & 6 & 4 & 1 & 2 & 0 & 2 & 1 & 2 & 2 & 6 & 4 \\
\hline 5 & 1 & 2 & 1 & 2 & 2 & 2 & 4 & 6 & 2 & 1 & 2 & 0 & 2 & 2 & 2 & 3 & 6 & 3 \\
\hline 6 & 2 & 2 & 0 & 2 & 2 & 2 & 4 & 6 & 2 & 2 & 2 & 1 & 2 & 2 & 2 & 5 & 6 & 1 \\
\hline 7 & 1 & 2 & 1 & 2 & 2 & 2 & 4 & 6 & 2 & 2 & 2 & 1 & 2 & 2 & 2 & 5 & 6 & 1 \\
\hline 8 & 2 & 2 & 2 & 2 & 0 & 1 & 4 & 5 & 1 & 1 & 2 & 2 & 2 & 0 & 1 & 3 & 5 & 2 \\
\hline 9 & 2 & 2 & 2 & 2 & 2 & 2 & 6 & 6 & 0 & 2 & 1 & 2 & 2 & 2 & 1 & 6 & 4 & -2 \\
\hline 10 & 2 & 2 & 0 & 2 & 2 & 2 & 4 & 6 & 2 & 1 & 2 & 1 & 2 & 2 & 2 & 4 & 6 & 2 \\
\hline$P$ & \multicolumn{2}{|r|}{.02} & \multicolumn{2}{|c|}{.009} & \multicolumn{2}{|r|}{.157} & \multicolumn{2}{|c|}{.007} & & \multicolumn{2}{|r|}{.03} & \multicolumn{2}{|c|}{.009} & \multicolumn{2}{|c|}{.564} & \multicolumn{2}{|c|}{.016} & \\
\hline
\end{tabular}

$\tau$ coefficient $=0.66, P=.018$.

tically significant agreement. Statistical analysis was performed using SPSS statistical software (Version 23.0; IBM, Armonk, New York).

\section{RESULTS \\ Patient Demographics/Presentations and mAVM Characteristics}

Patients were nearly equivalent in sex ( 6 females, 4 males) with a mean age of 43.5 years (range, 10-69 years) at the time of presentation. Neurologic deficits were noted in $90 \%$ of patients, and half $(50 \%)$ had headaches. Eight patients $(80 \%)$ presented with intracranial hemorrhage, 1 patient $(10 \%)$ had cerebellar/thalamic infarction, and a pediatric patient had altered mental status. All intracranial hemorrhage cases were intraparenchymal, except 1 with isolated intraventricular hemorrhage, with a mean volume of $20.3 \mathrm{~mL}$ (range, $0.5-55.5$ $\mathrm{mL})$. Intracranial hemorrhages were supratentorial in $5 / 8$ $(62.5 \%)$ patients and infratentorial in $3 / 8$ (37.5\%) patients. The On-line Table details patient demographics, clinical presentations, intracranial hemorrhage location/volume, mAVM classification/anatomy, and management/treatment.

CTA was performed at presentation in 3 patients, suggesting the possibility of a "small AVM" in patients 2 and 4 but was unremarkable in patient 10. MR imaging and MRA were performed for all patients at presentation; findings were questionable for an AVM in patient 4, while there was no report of an AVM in the remainder. DSA and IA-CBCTA were required for Spetzler-Martin grading of mAVMs that ranged from SpetzlerMartin grade I (40\%), Spetzler-Martin grade II (40\%), or Spetzler-Martin grade III (20\%) due to deep venous drainage and/or eloquent location in 6/10 (60\%). Most mAVMs, 7/10 $(70 \%)$, consisted of a very small micronidus of $<5 \mathrm{~mm}$. Only a single flow-induced aneurysm arising from a splenial branch feeder of the pericallosal anterior cerebral artery was identified (patient 4).

Management of mAVMs included microsurgical resection in 6 patients, stereotactic radiosurgery in 2 patients, and conservative surveillance in the remaining 2 patients. No endovascular embolization procedures were performed. When intervention was planned, the IA-CBCTA imaging data for mAVM localization was imported into the neuronavigation software for intraoperative guidance (iPlan 3.0 Cranial; Brainlab,
Munich, Germany) or treatment-planning software (Leksell GammaPlan 10; Elekta, Stockholm, Sweden) for stereotactic radiosurgery.

\section{IA-CBCTA Diagnostic and Treatment-Planning Observer Studies}

Blinded observer analysis was performed on DSA and multiplanar IA-CBCTA images from 10 patients with suspected intracranial mAVMs (Table 1). Initial DSA evaluation revealed a micronidus with excellent visibility in only 2 patients $(20 \%)$ per both observers (patients 8 and 9). In contrast, IA-CBCTA clearly identified the micronidus in all 10/10 (100\%) patients, with both observers scoring the nidus as grade 2 (excellent visualization) with perfect interobserver agreement. Interobserver agreement in grading the rest of the individual anatomic parameters with IA-CBCTA or DSA ranged from good to excellent ( $\tau$ coefficient between 0.55 and 1.0). Interobserver agreement for overall diagnostic value scores of IA-CBCTA and DSA was good $(\tau$ coefficient $=0.57$ and 0.7 , respectively).

Both neuroradiologists assigned significantly higher scores to IA-CBCTA for overall diagnostic value (both observers: $P<.05$; observer $1: P=.007$; observer $2: P=.016$ ), confirming the relative diagnostic value of IA-CBCTA. Although no significant differences were seen between DSA and IA-CBCTA scores when evaluating venous drainage (observer $1: P=.157$; observer 2 : $P=$ $.564)$, both observers assigned significantly higher scores to IACBCTA when evaluating arterial feeders (observer $1: P=.02$; observer 2: $P=.03$ ) and the micronidus (observer 1: $P=.009$; observer 2: $P=.009$ ), resulting in relative IA-CBCTA diagnostic value scores with good interobserver agreement $(\tau$ coefficient $=$ $0.66, P=.018)$.

Both neurosurgeons recorded more confidence in the treatment plan (grades 1-2) for nearly all patients after review of the IA-CBCTA and agreed that it provided better delineation and precise anatomic localization of the micronidus (Table 2). For example, IA-CBCTA led to both neurosurgeons altering their treatment plan (grade 2) in patient 1 (Fig 1, where the nidus could only be identified on IA-CBCTA) and recommending superselective catheterization and possible embolization before proceeding to an operation (grade 1) in patient 4 . Overall, they reported that IA-CBCTA would increase confidence in their microsurgical or radiosurgery treatment by importing these data directly into a 
Table 2: Treatment-planning scoring of IA-CBCTA relative to DSA ${ }^{a}$

\begin{tabular}{|c|c|c|c|c|c|c|}
\hline \multirow[b]{2}{*}{ Patient } & \multicolumn{2}{|c|}{ Neurosurgeon 1} & \multirow{2}{*}{$\begin{array}{l}\text { Relative CBCTA } \\
\text { Treatment Value }\end{array}$} & \multicolumn{2}{|c|}{ Neurosurgeon 2} & \multirow{2}{*}{$\begin{array}{l}\text { Relative CBCTA } \\
\text { Treatment Value }\end{array}$} \\
\hline & DSA & СВСТА & & DSA & СВСТА & \\
\hline 1 & Not sufficient & Operation & 2 & Not sufficient & Operation & 2 \\
\hline 2 & Radiosurgery & Radiosurgery & 1 & Radiosurgery & Radiosurgery & 1 \\
\hline 3 & Radiosurgery & Radiosurgery & 1 & Radiosurgery & Radiosurgery & 0 \\
\hline 4 & Operation & Operation & 1 & Operation & Operation & 1 \\
\hline 5 & Operation & Operation & 1 & Operation & Operation & 1 \\
\hline 6 & Radiosurgery & Radiosurgery & 1 & Radiosurgery & Radiosurgery & 1 \\
\hline 7 & Operation & Operation & 1 & Operation & Operation & 1 \\
\hline 8 & Radiosurgery & Radiosurgery & 1 & Radiosurgery & Radiosurgery & 1 \\
\hline 9 & Operation & Operation & 1 & Operation & Operation & 1 \\
\hline 10 & Operation & Operation & 1 & Operation & Operation & 1 \\
\hline
\end{tabular}

${ }^{\mathrm{a}} \tau$ coefficient $=0.73, P=.025$.

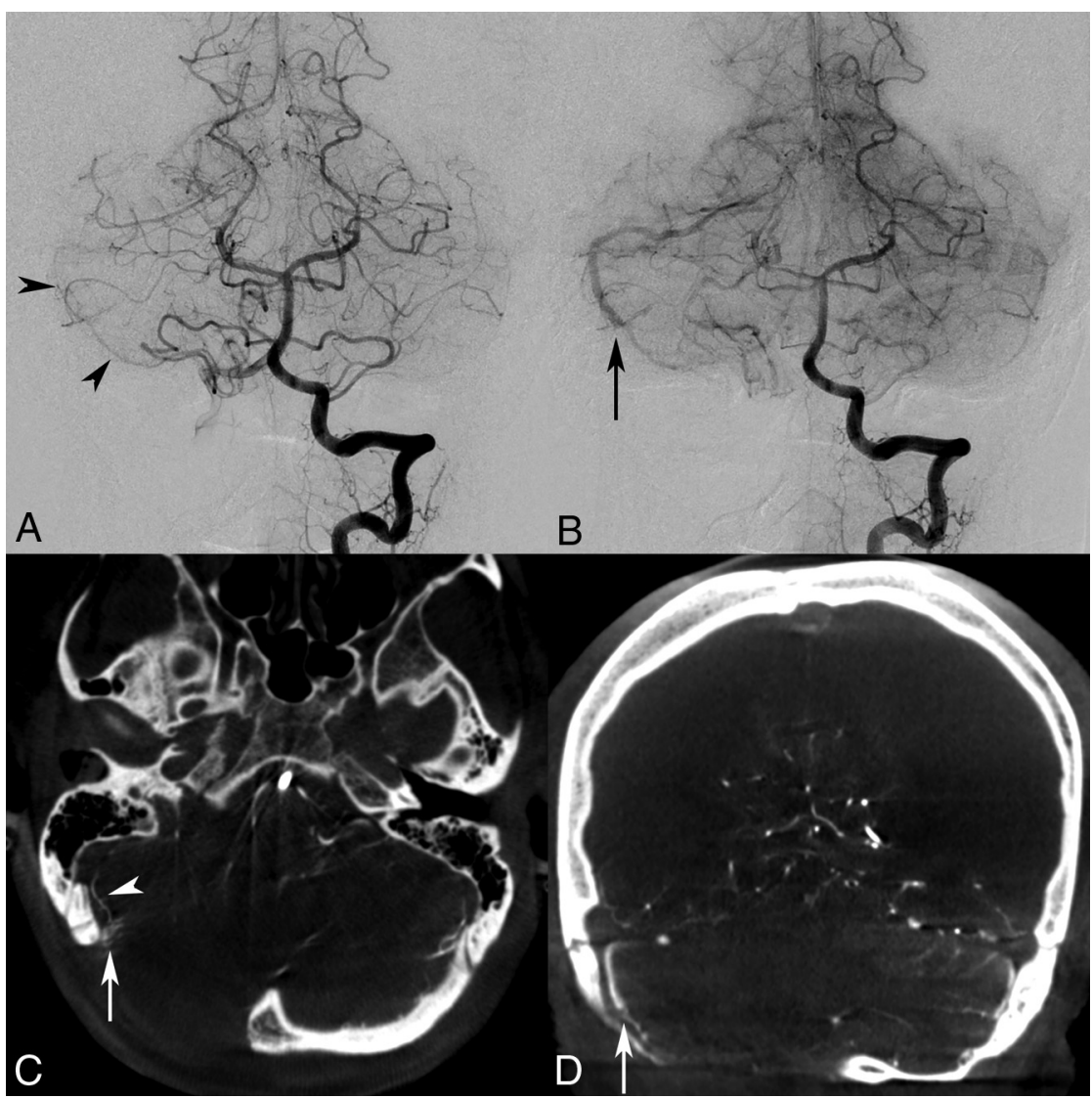

FIG 1. Anteroposterior DSA images demonstrate left vertebral artery injections in the early $(A)$ and late $(B)$ arterial phases, identifying arteriovenous shunting and early venous drainage into a hypertrophied superior cerebellar hemispheric vein (arrow) with tentorial venous outflow, but both observers were unable to appreciate an occult micronidus. Although arterial feeders are suggested to project into this region on DSA from the right anterior cerebellar artery and SCA ( $A$, arrowheads), the SCA feeder is better appreciated on IA-CBCTA multiplanar axial reconstructions ( $C$, arrowhead). Moreover, both observers identified a $<5$-mm micronidus under the lateral cerebellar surface and adjacent to the craniectomy site (C, arrow) with adjunctive IA-CBCTA reconstructions, consistent with an $\mathrm{MAVM}$. IA-CBCTA coronal reconstruction $(D)$ shows the nidal outflow to the early draining superior cerebellar hemispheric vein (arrow).

neuronavigation system. Interobserver agreement between the 2 vascular neurosurgeons for the relative IA-CBCTA treatmentplanning value was good $(\tau$ coefficient $=0.73, P=.025)$.

\section{Illustrative Cases}

Patient 1. A 30-year-old woman presented with a 3-year history of epilepsy, periventricular heterotopia was diagnosed with a pre- sumed right cerebellar developmental venous anomaly after emergent outside imaging evaluation including DSA. Due to acute presentation with severe headache, visual impairment, quadriparesis, and rapid deterioration to unconsciousness, she required emergent craniectomy and decompression of a cerebellar parenchymal hemorrhage. Although initial MR imaging/MRA failed to detect a vascular malformation, a 3-month delayed DSA demonstrated findings suspicious for a pial AVF (Fig 1A,B). At 6 months, repeat DSA with 3DRA redemonstrated arteriovenous shunting and early venous drainage but also identified an occult $<5$-mm micronidus under the lateral right cerebellar surface, adjacent to the craniectomy site, and only visualized with IA-CBCTA reconstructions (Fig 1C, D).

Patient 4. A 21-year-old woman presented to our institution with sudden headache and dizziness for 1 hour. Initial CT/CTA and MR imaging/MRA brain studies revealed an acute paramedian right frontoparietal intracerebral hemorrhage and a distal pericallosal aneurysm with suspicion of an underlying occult vascular malformation, but no abnormal vascular flow voids or nidus was seen (Fig 2A). Subsequent DSA with the 3DRA/IA-CBCTA technique was performed 2 days later confirming a 2-mm pericallosal-splenial artery aneurysm/pseudoaneurysm with suggestion of an early draining vein (Fig $2 B, C$ ), but a definitive arterial feeder and $<5$-mm nidus were only visualized on IA-CBCTA multiplanar reconstructions to confirm the diagnosis of a micro-AVM (Fig 2D-G).

Patient 9. A 50-year-old man presented to an outside institution with confusion followed by right hemiparesis. Initial CT/MR imaging discovered a left parietal cortical hemorrhage, which was 

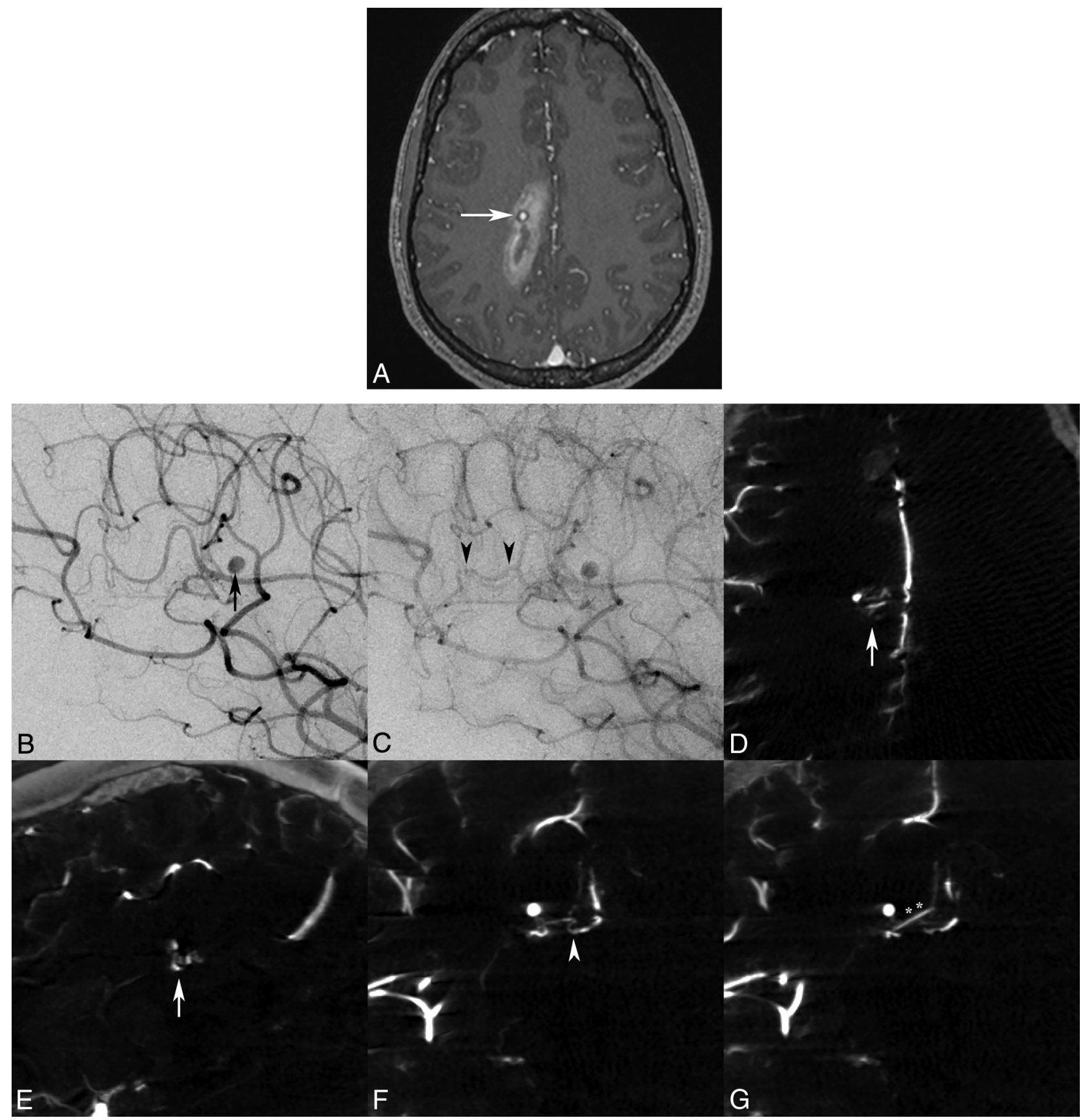

FIG 2. Axial MR imaging MPRAGE postgadolinium $(A)$ image demonstrates a right parasagittal frontoparietal intraparenchymal hemorrhage with a contrast-enhancing pseudoaneurysm (white arrow), consistent with the rupture site. Lateral oblique DSA images in the early arterial phase confirm a pericallosal anterior cerebral artery aneurysm/pseudoaneurysm ( $B$, black arrow), with a subtle early draining vein in the capillary phase (C, black arrowheads), but no distinct vascular nidus was identified by either observer. Only IA-CBCTA multiplanar reconstructions clearly delineate a $<5-\mathrm{mm}$ micronidus on axial and sagittal reconstructions ( $D$ and $E$, white arrows). Coronal multiplanar reconstructions also assist in identification of the small arterial feeder from the pericallosal-splenial artery branch ( $F$, white arrowhead) and single draining vein (G, double asterisks) directly associated with the micronidus and flow-induced pseudoaneurysm.

conservatively managed. Delayed 2-month MR imaging findings were negative, but subsequent DSA reported a left parietal pial AVF, and embolization was planned. At our institution, a repeat DSA with the 3DRA/IA-CBCTA technique revealed a superficial left parietal $\mathrm{mAVM}$ with a nidus measuring $<1 \mathrm{~cm}$ (Fig $3 A, B$ ). Left frontoparietal paramedian craniotomy and microsurgical resection were performed with IA-CBCTA imaging integrated into the neuronavigation system (Fig $3 C, D$ ).

\section{DISCUSSION}

Prior literature emphasized the importance of identifying an $\mathrm{mAVM}$ and characterizing its anatomy for effective treatment. However, the diagnosis of mAVMs can be challenging. Alén et al ${ }^{3}$ reported the role of high-resolution MR imaging fast spin-echo $\mathrm{T} 2$-weighted sequences in detecting subtle flow voids corresponding to small nidus volumes. In addition, they noted the supplementary value of contrast-enhanced MR imaging/MRA over 


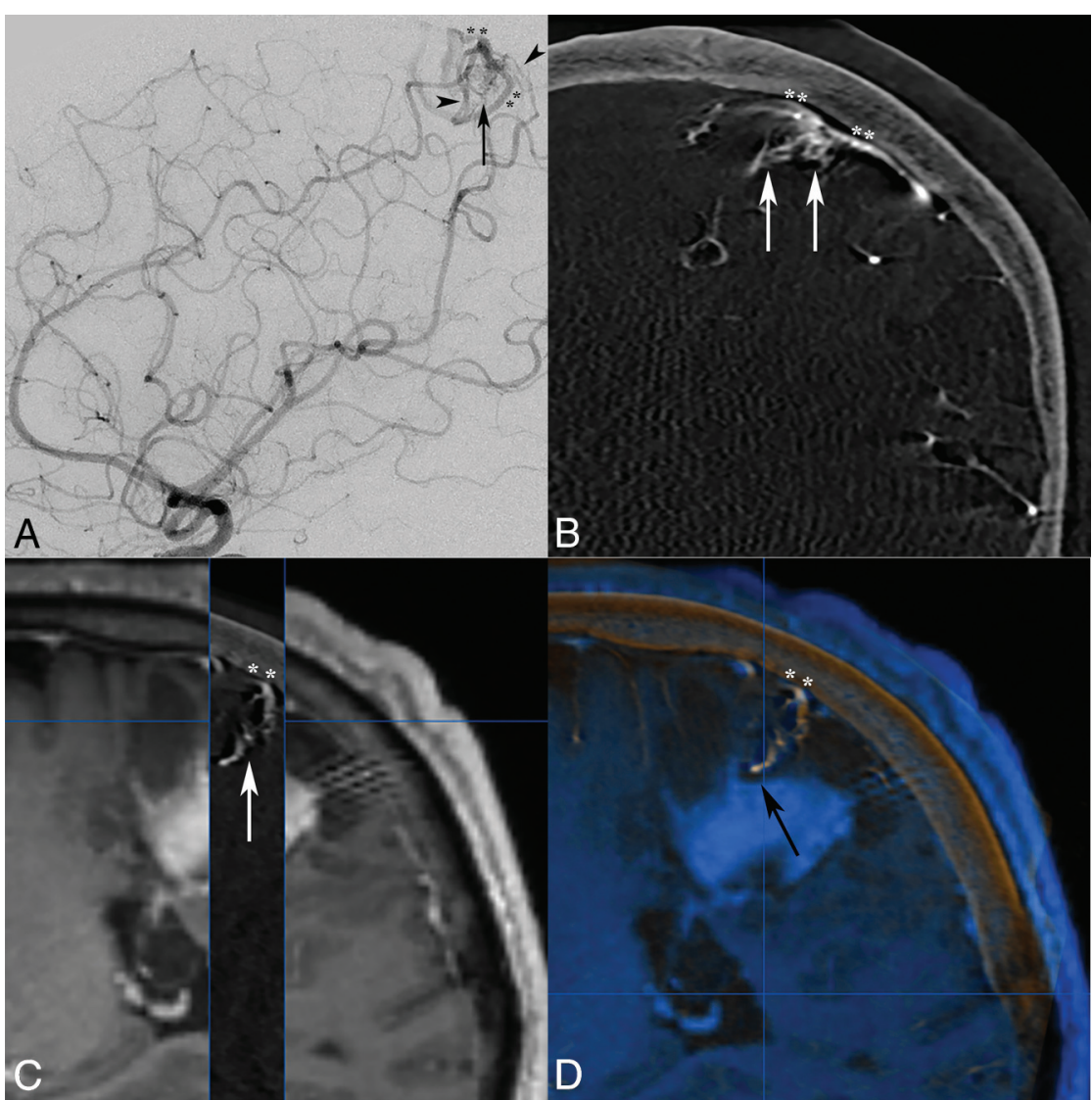

FIG 3. Lateral DSA $(A)$ and coronal IA-CBCTA reconstruction $(B)$ images both demonstrate a small $<\mathrm{l}$-cm micronidus (arrows), supplied by tortuous parietal branches of the pericallosal anterior cerebral artery and inferior division of the MCA (arrowheads), with early venous drainage into bifurcating cortical veins (double asterisks). Although both observers did not report the improved diagnostic value of IA-CBCTA in this case, both neurosurgeons reported increased confidence in treatment planning, and IA-CBCTA was incorporated into the neuronavigation system for microsurgical resection. 3D IA-CBCTA and MR imaging datasets were merged with sagittal overlay (C) delineating the micronidus (arrow) and draining cortical vein (double asterisks) complex in relation to the adjacent hemorrhage. Both datasets were imported into the intraoperative neuronavigation system $(D, B$ rain $L A B)$, allowing anatomic localization of the micronidus within a specific sulcus guiding the surgical approach as well as the presumed deep nidal rupture site abutting the hematoma (black arrow).

time-of-flight MRA techniques for nidus delineation (especially when adjacent to a hematoma, due to background suppression of hyperintense methemoglobin). However, even combined MR imaging/MRA techniques failed to identify mAVMs in 6/21 patients, and they required DSA to confirm the diagnosis. Early DSA in the setting of hemorrhagic mass effect may also miss mAVMs secondary to diminished flow from various causes such as hemorrhage-related vasospasm, compression of the nidus or feeder/draining vessel, or intralesional thrombosis. ${ }^{11}$ Some have reported initially negative DSA findings in patients with hemorrhage who were proven later to have an $\mathrm{mAVM}$ on histopathology or repeat angiography, ${ }^{3,5,11}$ leading other authors to propose that negative or questionable conventional DSA findings in young adults with atypical hemorrhage patterns should prompt superselective angiography and/or a repeat study after hemorrhage resorption. ${ }^{2,3,11,12}$

In our series, even $2 \mathrm{D}$ and 3DRA techniques without IACBCTA reconstructions were relatively inferior in delineating the AVM arterial feeders and nidus, consistent with mAVMs possibly being very small occult lesions $(<5 \mathrm{~mm})$. We have shown that
3DRA with IA-CBCTA multiplanar reconstructions increases diagnostic power, enabling angiographic identification and precise localization of occult mAVMs over traditional 2D DSA techniques. IA-CBCTA techniques may thereby limit the need for superselective catheterization and repeat angiography. Additionally, while conventional DSA still maintains superior spatial and temporal resolution to diagnose arteriovenous shunting or an abnormal early draining vein, it has some limitations due to its 2D acquisition and inherent vessel overlap, which may interfere with arterial feeder and micronidus delineation. ${ }^{9}$ In contrast, the precise cross-sectional localization of mAVMs, especially for surgical treatment planning, can be readily facilitated by IA-CBCTA.

Our results are in keeping with previous studies that have shown the feasibility and superiority of using IACBCTA in both preoperative planning and as an intraoperative reference for the microsurgical resection or stereotactic radiosurgery of larger brain AVMs. ${ }^{13-15}$ In a case series of 16 patients with cerebral AVMs, Srinivasan et al ${ }^{14}$ used CBCTA in a neuronavigation system, allowing complete and safe surgical resection of all lesions. In another case series by Safain et $\mathrm{al}^{15}$ of 22 patients, CBCTA was used to target cerebral AVMs with radiosurgery, with only $4 / 22$ lesions that had a micronidus of $1 \mathrm{~cm}$ and resulted in improved visualization of AVM components and treatment planning. Radvany et al ${ }^{16}$ described a modified CBCTA imaging technique in 3 patients performed using an intra-arterial diluted iodinated contrast agent (35\%) in the ascending aorta that lasted 22 seconds at a rate of $8 \mathrm{~mL} / \mathrm{s}$, which allows imaging of an entire AVM nidus receiving blood supply from $>1$ cervical artery in a single CBCTA acquisition. In this study, our intra-arterial technique was modified with shorter acquisition times (5-8 seconds) to avoid venous contamination and single cervical artery infusion of nondiluted contrast to optimize visualization of the micronidus and early draining vein. Rahal and Malek ${ }^{17}$ reported a higher sensitivity of IA-CBCTA over DSA in identifying the occult nidal anatomy and angioarchitecture of brain AVMs in 3 patients presenting with intracranial hemorrhage as well providing anatomic guidance for either surgical or radiosurgery treatment. However, none of these studies included or discussed the value of CBCTA in cerebral AVMs with a micronidus of $<1 \mathrm{~cm}$.

Additionally, IA-CBCTA has been shown to improve the anatomic delineation and localization of both intracranial and spinal dural arteriovenous fistulas for preoperative or endovascular 
treatment planning. ${ }^{8,9,18}$ Flat panel detectors improve the signal intensity-to-noise ratio and suppress geometric distortion, enabling 3DRA and IA-CBCTA to produce high spatial and contrast-resolution imaging with radiation and contrast-dose reduction. ${ }^{19}$ Isotropic CBCT volumetric datasets allow multiplanar and $3 \mathrm{D}$ reconstructions analogous to multidetector CT scanners. ${ }^{9}$ Advantages over traditional intravenous CT angiography include submillimeter-spatial-resolution isotropic data with versatile postprocessing software analysis, selective intra-arterial contrast injections for territorial vascular analysis, rapid and variable acquisitions (5-8 seconds) to optimize early venous opacification versus venous contamination, subtraction and hemodynamic flow analysis (3DRA), and unsubtracted cross-sectional localization relative to the osseous structures and soft tissues (IA-CBCTA). ${ }^{9,14}$

Our study is subject to the inherent limitations of a retrospective series and qualitative grading of DSA images in comparison with IA-CBCTA as an adjunctive imaging technique. We attempted to partly mitigate this bias with blinded observer analysis and a protocol for grading DSA and IA-CBCTA imaging in series on a de-identified workstation that retained the ability to analyze IA-CBCTA multiplanar reconstructions with or without tissue subtraction. Furthermore, memory bias was limited, with an elapsed period of 6 years to accumulate our cases and only 2 observers in each arm of the study from a total of 6 neurointerventionalists involved in the initial diagnostic evaluation and 5 vascular neurosurgeons involved in the surgical/radiation treatment. Due to the rare incidence of mAVMs, sample sizes in all studies of this pathology remained small, and future studies with a larger population would require a multi-institutional cohort or registry.

\section{CONCLUSIONS}

We compared DSA and adjunctive IA-CBCTA imaging to assess the relative efficacy of IA-CBCTA in the diagnosis and treatment planning of mAVMs. IA-CBCTA improves the diagnostic accuracy of mAVMs, particularly through enhanced anatomic identification and localization of subtle arterial feeders and/or a micronidus, and enables more confident surgical/radiosurgery treatment planning.

\section{ACKNOWLEDGMENTS}

We thank Ms Saadia Sattar, MSc, who contributed to the statistical analysis of this study.

Disclosures: Sameer A. Ansari—UNRELATED: Grants/Grants Pending: MicroVention postdoctoral research training grant.* * Money paid to the institution.

\section{REFERENCES}

1. Stiver SI, Ogilvy CS. Micro-arteriovenous malformations: significant hemorrhage from small arteriovenous shunts. Neurosurgery 2000;46:811-18; discussion 818-19 Medline

2. Andreou A, Ioannidis I, Lalloo S, et al. Endovascular treatment of intracranial microarteriovenous malformations. J Neurosurg 2008; 109:1091-97 CrossRef Medline
3. Alén JF, Lagares A, Paredes I, et al. Cerebral microarteriovenous malformations: a series of 28 cases. J Neurosurg 2013;119:594-602 CrossRef Medline

4. Stiver SI. Microarteriovenous malformations. Neurosurg Clin N Am 1999;10:485-501 Medline

5. Willinsky R, Lasjaunias P, Comoy J, et al. Cerebral micro arteriovenous malformations (mAVMs): review of 13 cases. Acta Neurochir (Wien) 1988;91:37-41 CrossRef Medline

6. Alexander E. Microneurosurgery. IIIA: AVM of the brain, history, embryology, pathological considerations, hemodynamics, diagnostic studies, microsurgical anatomy: edited by M. G. Yasargil. 408 pages. New York: Georg Thieme Verlag, 1987 (book review). Surg Neurol 1988;29:494

7. Wallace MJ, Kuo MD, Glaiberman C, et al; Technology Assessment Committee of the Society of Interventional Radiology. Three-dimensional C-arm cone-beam CT: applications in the interventional suite. J Vasc Interv Radiol 2009;20:S523-37 CrossRef Medline

8. Honarmand AR, Gemmete JJ, Hurley MC, et al. Adjunctive value of intra-arterial cone beam CT angiography relative to DSA in the evaluation of cranial and spinal arteriovenous fistulas. J Neurointerv Surg 2015;7:517-23 CrossRef Medline

9. Aadland TD, Thielen KR, Kaufmann TJ, et al. 3D C-arm conebeam $\mathrm{CT}$ angiography as an adjunct in the precise anatomic characterization of spinal dural arteriovenous fistulas. AJNR Am J Neuroradiol 2010;31:476-80 CrossRef Medline

10. Luby M, Hong J, Merino JG, et al. Stroke mismatch volume with the use of $\mathrm{ABC} / 2$ is equivalent to planimetric stroke mismatch volume. AJNR Am J Neuroradiol 2013;34:1901-07 CrossRef Medline

11. Elhammady MS, Baskaya MK, Heros RC. Early elective surgical exploration of spontaneous intracerebral hematomas of unknown origin. J Neurosurg 2008;109:1005-11 Medline

12. Willinsky R, TerBrugge K, Montanera W, et al. Micro-arteriovenous malformations of the brain: superselective angiography in diagnosis and treatment. AJNR Am J Neuroradiol 1992;13:325-30 Medline

13. van der Bom IM, Gounis MJ, Ding L, et al. Target delineation for radiosurgery of a small brain arteriovenous malformation using high-resolution contrast-enhanced cone beam CT. J Neurointerv Surg 2014;6:e34 CrossRef Medline

14. Srinivasan VM, Schafer S, Ghali MG, et al. Cone-beam CT angiography (DynaCT) for intraoperative localization of cerebral arteriovenous malformations. J Neurointerv Surg 2016;8:69-74 CrossRef Medline

15. Safain MG, Rahal JP, Raval A, et al. Use of cone-beam computed tomography angiography in planning for gamma knife radiosurgery for arteriovenous malformations: a case series and early report. Neurosurgery 2014;74:682-95; discussion 695-96 CrossRef Medline

16. Radvany MG, Ehtiati T, Huang J, et al. Aortic arch injection with C-arm cone beam CT for radiosurgery treatment planning of cerebral arteriovenous malformations: technical note. J Neurointerv Surg 2012;4:e28 CrossRef Medline

17. Rahal JP, Malek AM. Benefit of cone-beam computed tomography angiography in acute management of angiographically undetectable ruptured arteriovenous malformations. J Neurosurg 2013;119: 1015-20 CrossRef Medline

18. Ansari SA, Aoun SG, Bendok BR. Cone beam computed tomography in the neurointerventional room: beyond vessels. World Neurosurg 2012;77:659-61 CrossRef Medline

19. Lai CJ, Shaw CC, Chen L, et al. Visibility of microcalcification in cone beam breast CT: effects of X-ray tube voltage and radiation dose. Med Phys 2007;34:2995-3004 CrossRef Medline 